\title{
Reviewer acknowledgment 2015
}

Hugo ten Cate ${ }^{1 *}$ and Yukio Ozaki ${ }^{2}$

\section{Contributing reviewers}

The Editors of Thrombosis Journal would like to thank all of our reviewers who have contributed to the journal in Volume 13 (2015) and whose valuable support is fundamental to its success.

\author{
Robert Ariens \\ UK \\ Juan Badimon \\ USA \\ Erik Beckers \\ Netherlands \\ Lars Borris \\ Denmark
}

Annemieke Bouman

Netherlands

Suzanne Cannegieter

Netherlands

\section{Mario M D'Elios \\ Italy}

\section{Matteo Nicola Dario Di Minno Italy}

\section{Marcello Di Nisio \\ Italy}

Jonathan Douxfils

Belgium

\section{William Fisher}

Canada

Satoshi Fujii

Japan

\author{
Alexander Gallus \\ Australia \\ Esteban Gandara \\ Canada
}

Hadi Goubran Messiha

Canada

José Govers-Riemslag

Netherlands

Nil Guler

Turkey

Karly Hamulyak

Netherlands

Yvonne Henskens

Netherlands

Masahiro Ieko

Japan

David Jiménez

Spain

Frederikus Klok

Netherlands

Takao Kobayashi

Japan

Amartya Kundu

USA
Seiji Madoiwa

Japan

Harry Magnani

Netherlands

Karina Meijer

Netherlands

Marco Moia

Italy

\section{Mashio Nakamura}

Japan

Marisa Ninivaggi

Netherlands

Michael Nurmohamed

Netherlands

Toshiyuki Ojima

Japan

Marina Panova-Noeva

Germany

Ron Pisters

Netherlands

Domenico Prisco

Italy

Jürgen Prochaska

Germany

* Correspondence: thrombosisjournal@biomedcentral.com

${ }^{1}$ Academic Hospital Maastricht, Maastricht, The Netherlands

${ }^{2}$ University of Yamanashi, Kofu, Japan 
Tomohiro Sakamoto

Japan

Masato Sakon

Japan

Carlos Santos-gallego

USA

Nobuyuki Sato

Japan

Craig Seaman

USA

Toshiro Shinke

Japan

Tomonori Sugiura

Japan

Hiroyuki Sumi

Japan
Hideyuki Takedani

Japan

Hiroki Tanaka

Japan

Arina Ten Cate-Hoek

Netherlands

Kazuo Umemura

Japan

Naoki Unno

Japan

Tom van der Hulle

Netherlands

Nick Van Es

Netherlands

Minka Vries

Netherlands
Jeanine Walenga

USA

Mark Walsh

USA

Theodore Warkentin

Canada

Siegfried Wieshammer

Germany

Kristien Winckers

Netherlands

Yi Wu

USA

Aizhen Yang

China

Urooj Zafar

USA 